\title{
Endovascular occlusion of intracranial aneurysms with electrically detachable platinum coils
}

\section{Pieter Fourie}

MBChB (Pret), MMed(Rad)Pret

Consultant Radiologist, Pretoria Heart Hospital

GDC coil

occlusion of

intracranial

aneurysms is a

new and rapidly

evolving

neurointerven-

tional technique.

The results of the

first South

African centre

are presented.

\section{Incidence}

The incidence of intracranial aneurysms varies from $1-2 \%{ }^{1}$ This is equal to about 10000 for every million people in a general population.

In 1989 alone some 40000 individuals with aneurysms were hospitalised in the USA. ${ }^{2}$ Sixty percent of these patients were treated conservatively because of poor medical conditions, advanced age and other factors. The mortality rate was approximately $60 \%$ and morbidity was $25-40 \%$ within 6 months after the original haemorrhage. ${ }^{3}$

Different morphological types exist. The saccular or berry aneurysms are sometimes referred to as congenital. Fusiform aneurysms have no neck and are usually atherosclerotic in origin. They are also seen in patients with medio-necrosis and Marfan Syndrome. Other causes for aneurysms include mycotic, dissecting and traumatic, and Moyamoya disease. ${ }^{4}$

\section{Aneurysm sizes and flow patterns}

Aneurysms are classified into:

Small $<10 \mathrm{~mm}$; medium 10-25mm and giant $>25 \mathrm{~mm}$ in diameter. Each aneurysm has a characteristic flow pattern and possiblecomplications.

Ferguson et al studied flow patterns in aneurysms in glass models ${ }^{5}$. Disturbed chaotic flow is described by Liepsch et al. ${ }^{6}$ This flow varies from laminar to turbulent. An inflow tract and outflow tract are now recognised with a high energy pressure gradient in the central part of the inflow tract.

Stasis of flow in giant aneurysms can be demonstrated with the deposition of leucocytes and fibrin causing thrombus formation. These very large aneurysms havelow shear stress on the aneurysm walls which leads to gradual enlargement. Smaller aneurysms may experience high wall stresses leading to easier rupture. ${ }^{8}$

\section{Diagnosis}

Most patients with aneurysms present with haemorrhage due to rupture. The primary purpose of treatment of ruptured aneurysms is to prevent an often fatal rebleed. Most ruptures occur at the dome of the aneurysm, but sometimes at the neck. Another important sequel of haemorrhage due to rupture is the onset of vasospasm. Early treatment of the aneurysm may lessen this complication.

The clinical presentation of the patient usually warrants further investigation such as $\mathrm{CT}$ and possibly MR to demonstrate haemorrhage with or without mass effect. (Figures la\&lb)

The size, position, neck length and the presence of intraluminal thrombus are important features that need close inspection before any treatment.

Three dimensional $\mathrm{CT}$ and $\mathrm{MR}$ are valuable tools in demonstrating the aneurysmal neck in relation to the parent 


\section{Endovascular occlusion of intracranial}

aneurysms with electrically detachable platinum coils

\section{from page 10}
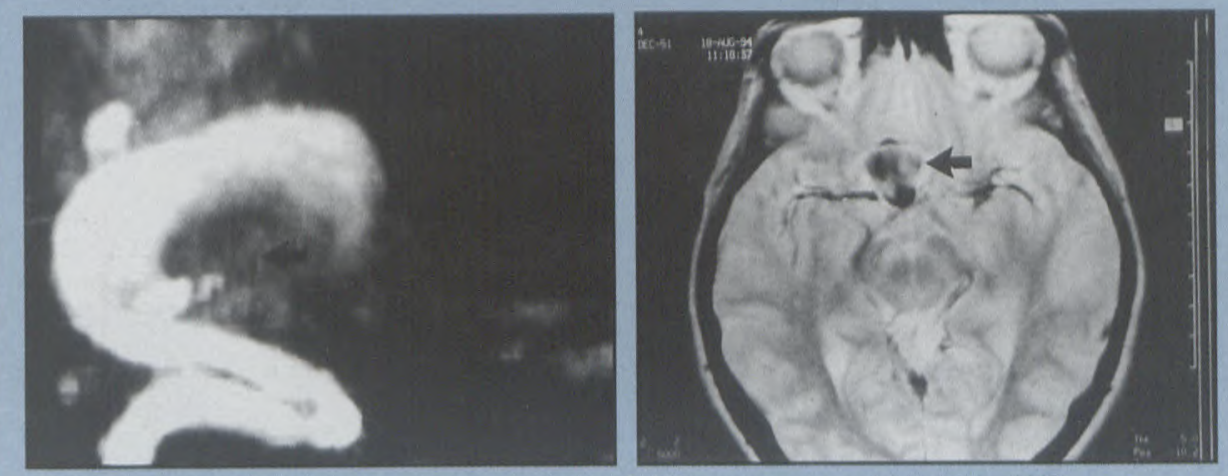

Figure 1: Intraluminal clot can be well demonstrated by (a)MRI angiography and (b)by normal MR.

vessel. Wide neck aneurysms are contraindicated for endovascular coiling as the coil will often displace into the parent vessel.(Figures 2a \&2b)
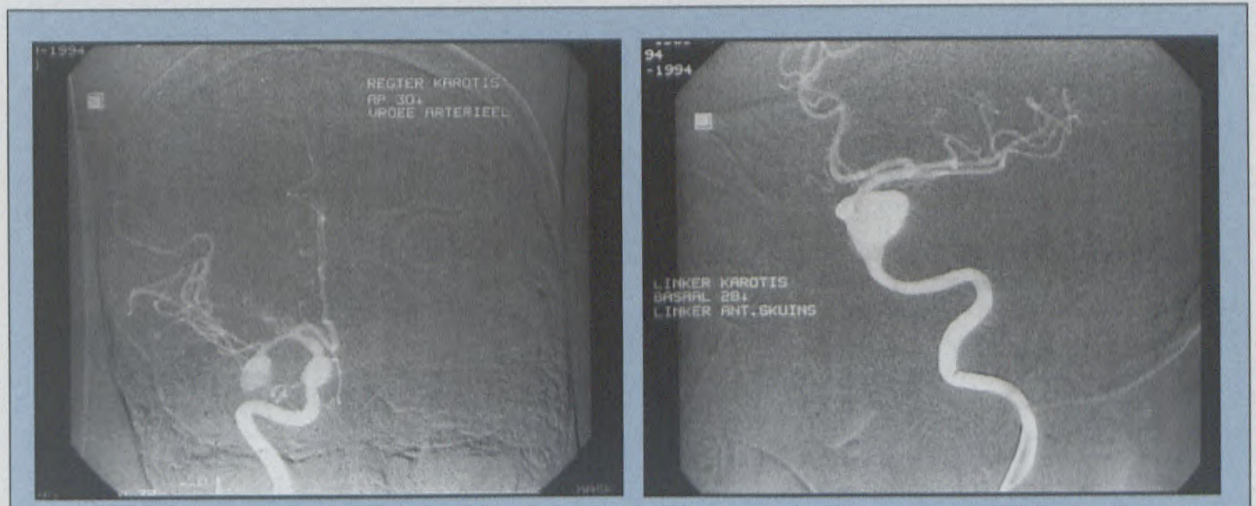

Figure 2: (a) A narrow neck aneurysm is better suited for GDC treatment than (b) the aneurysm with the broad base which was contraindicated for GDC in this case.

The presence of intraluminal thrombus may enhance coil compression and should be known prior to the treatment. Multiple aneurysms must be demonstrated if present. It is a well known fact that smaller aneurysms tend to rupture more easily than thelarger slow flow types, and should be treated first.(Figure3)

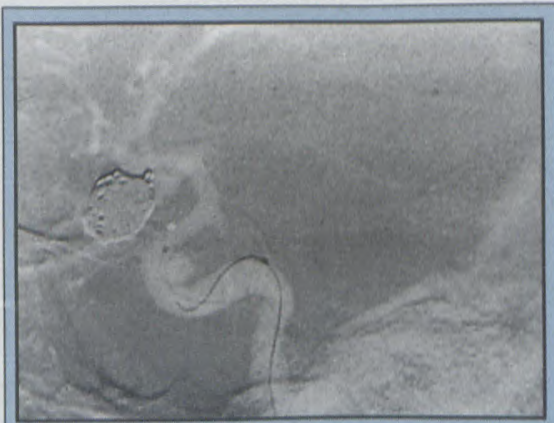

Figure 3: The larger aneurysm packed full of GDC coils has a narrow neck and is well suited for GDC treatment. The smaller one proximally has a broad base and was not treated with GDC.

\section{Treatment}

The surgical approach involves craniotomy, brain retraction and vessel manipulation with its possible complications.
The endovascular approach is a possible alternative for the treatment of certain aneurysms. The saccular aneurysms with relatively narrow necks fall into this group.

In 1991 Guglielmi et al reported on 15 cases with aneurysms treated via the endovascular route with detachable platinum coils. Since then some 4000 patients in other countries and 1300 in the USA

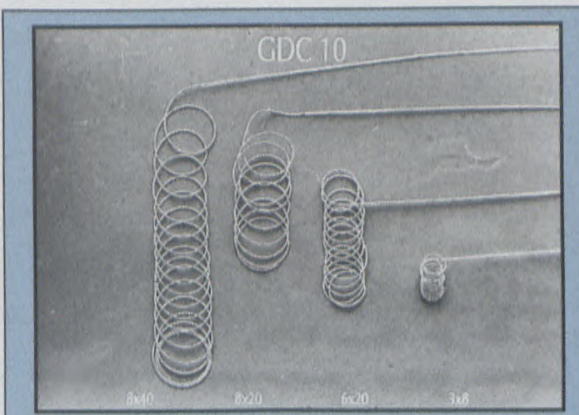
the soldered areas can also be seen. were treated with Guglielmi detachable coils(GDC).

Indications for treatment with GDC include:

- Location of the aneurysm (unsuitable surgical approach)

- Anticipated technical difficulties during surgery

- Previous surgical failure

- Patient refusal of surgery

The general use of GDCs in the USA was only allowed in early 1996 by the Food and DrugAdministration after a five year trial period.

\section{Guglielmi detachable coils (by Target therapeutics, California)}

The GDC consists of a platinum core wire with a diameter of $0,00175 "-0,004$ ". The core wire is rolled in a tight mesh forming a single strand of $0,0095 "-0,015$ " in diameter. These platinum wires are extremely soft and pliable. Four different diameters of wire exist from 0,0095 " to the so called soft wire, and 0,010 " or so called GDC 10 and $0,015^{\prime \prime}$ or so called GDC 18. A soft wire in the GDC 18 is also now manufactured and measures 0,0135 "in diameter.

These platinum wires are soldered to a stainless steel feeding wire. (Figures $4 \mathrm{a} \& 4 \mathrm{~b}$ ) The platinum wire is curled into a circular memory and stored in a plastic tubing for protection during transport. The circular memories available vary from $2-20 \mathrm{~mm}$ in

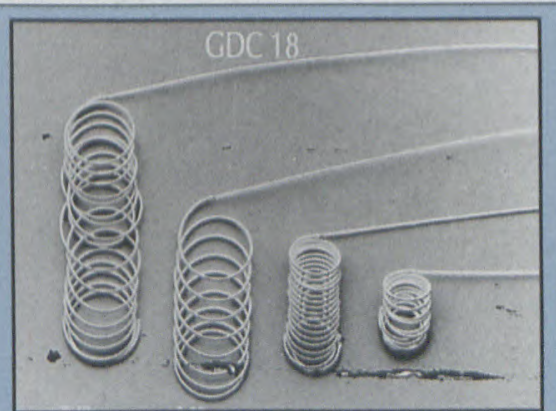

Figure 4a:Guglielmi detachable coil (GDC)10 coil. (b) Guglielmi detachable coil (GDC) 18 coil.

The memory curl can be seen. The lengths of the wires differ. The stainless steel wires are on the right and 


\section{from page 11}

diameter. These are made with the view of filling as close as possible the inside diameter of any given aneurysm up to $20 \mathrm{~mm}$ in size. Larger aneurysms can be embolised by these as well. (Figures $5 \mathrm{a} \& 5 \mathrm{~b}$ ).

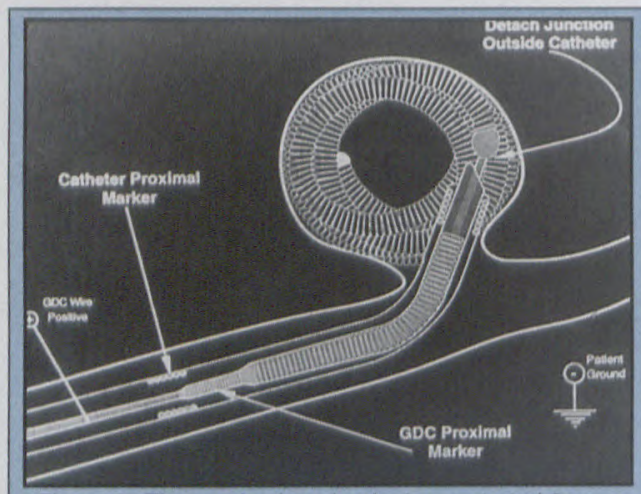

Figure 5a:The proximal marker is demonstrated $3 \mathrm{~cm}$ from the tip. When the proximal marker on the catheter and wire overlap the soldered area is just outside the catheter tip and can be detached.

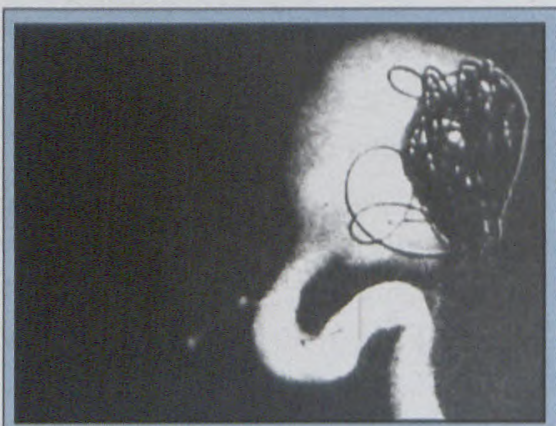

Figure 5b: The two markers can be seen in this picture. The first few coils have been released into the aneurysm.

The soldered area is destroyed by means of electrolysis when an electrical current of $1-2 \mathrm{~mA}$ from a battery operated generator is applied to the stainless steel wire. The smaller the diameter of thememory the shorter the wire. Wirelengths vary from $4-30 \mathrm{~cm}$.

\section{Team work}

Our team consists of a neurosurgeon, neurointerventional radiologist and anaesthetist. Theneurosurgeon heads the team and cares for the patient clinically and is usually present in the theatre for some time during the procedure. The possible efficiency of proposed treatment is discussed on a team basis and shared with the patient and the family.

\section{Possible complications due to instrumentation}

1 Vessel spasm during catheter manipulation is a well known problem in the best of hands. The large distal intracerebral arteries may undergo spasm. This problem is seen especially where tortuous vessels need to be negotiated by the catheter.

2 Vessel and/or aneurysm perforation can occur especially where fresh thrombus covers a recent ruptured aneurysm dome. The sudden forward movement of a microcatheter can be dangerous and should be anticipated.A slow outwards movement of the catheter may negate this problem.

3 A backward movement of the delivery system may pose a problem during advancement of the coil into the aneurysm.

4. During the removal of a wrongly placed coil, unravelling of the basket into the parent vessel may occur. Great care should be taken whenever a coil is removed. Elongation of the coil being removed is also a problem and the coil should be removed intermittently with great care.

5 Whenever aneurysm perforation has occurred the aneurysm should be packed with coils as soon as possible and Heparin reversed with protamine sulphate.

6. Distal embolism with possible stroke.

7. Possible parent vessel obstruction in wideneck aneurysms.

With the dangers of possible thromboembolism we keep our patients heparinized for 24 hours. TheACT is kept between 250-300 seconds. The sheath is only removed after 24 hours, when the ACT has dropped to 150 seconds.

\section{Clinical materials and methods}

Over aperiod of 18 months 21 patientswith cerebral aneurysms were seen, of which 9 were treated with GDCs. Nine were male and 12 were female. Their ages varied from 35-82 years. The female average age was 53 and that of the male 46.5 years.

The group anatomical positions of the aneurysms are depicted in Figure 6.

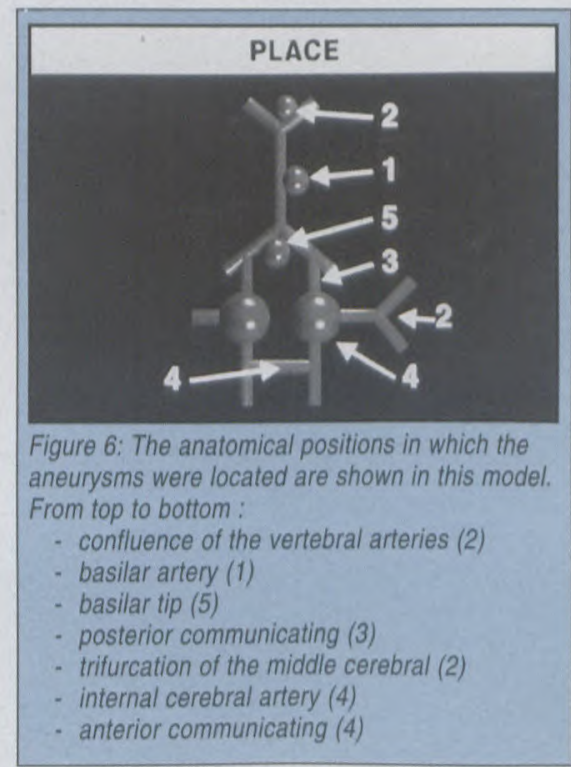

Sizes: $\quad 2$ weresmall, $<10 \mathrm{~mm}$

10weremedium, $10-25 \mathrm{~mm}$

9 were giant, $>25 \mathrm{~mm}$

According to Hunt \& Hess classification: ${ }^{10}$

7 were Hunt \& Hess I

12 wereHunt\&Hess II

2 were Hunt \& Hess III

Of the 9 giant aneurysms, 7 were treated and packed to $\pm 85 \%-95 \%$. We felt that the medium sized aneurysms were better packed than thelarger ones. (TableI)

\section{Follow-up}

Five cases did not have follow-up angiograms. Some were not available and were lost for follow-up, while some are still due for follow-up investigations.

Of the 12 patients that came for followup angiograms 8 had coil compaction. All the giant aneurysms demonstrated coil compaction while of the 5 medium sized aneurysms, 3 had compaction. One small aneurysm had no compaction. 


\section{Endovascular occlusion of intracranial aneurysms with electrically detachable platinum coils}

from page 12

\begin{tabular}{|c|c|c|c|c|c|c|}
\hline Patient & Age & Place & Size & $\mathrm{H} \& \mathrm{H}$ & Packed & Follow-up \\
\hline 1 & 44 & Ant.communis & giant & $\|$ & $\pm 95 \%$ & coil compaction \\
\hline 2 & 42 & Basilar base & medium & $\|$ & $\pm 95 \%$ & no compaction \\
\hline 3 & 46 & Int.carotid & giant & III & $\pm 95 \%$ & coil compaction \\
\hline 4 & 43 & Ant.communis & giant & II & $\pm 90 \%$ & coil compaction \\
\hline 5 & 40 & Trifurcation & medium & II & $\pm 95 \%$ & died \\
\hline 6 & 46 & Int.carotid & giant & 1 & not done & - \\
\hline 7 & 42 & Basilar base & medium & II & $\pm 80 \%$ & died \\
\hline 8 & 60 & Basilar base & medium & III & $\pm 90 \%$ & OK \\
\hline 9 & 59 & Int.carotid & giant & II & not done & - \\
\hline 10 & 46 & Ant.communis & medium & 1 & $\pm 90 \%$ & no follow-up \\
\hline 11 & 82 & Basilar tip & medium & ॥ & $\pm 95 \%$ & no follow-up \\
\hline 12 & 47 & Ophthalmic & giant & ॥ & $\pm 95 \%$ & coil compaction \\
\hline 13 & 55 & Basilar tip & giant & ॥ & $\pm 95 \%$ & coil compaction \\
\hline 14 & 29 & Trifurcation & small & 1 & $\pm 95 \%$ & no compaction \\
\hline 15 & 61 & Post.communis & medium & 1 & $\pm 95 \%$ & no compaction \\
\hline 16 & 55 & Post.communis & giant & ॥ & $\pm 95 \%$ & no follow-up \\
\hline 17 & 50 & Basilar tip & medium & ॥ & $\pm 95 \%$ & slight compaction \\
\hline 18 & 44 & Basilar tip & medium & 1 & $\pm 90 \%$ & coil compaction \\
\hline 19 & 67 & Post.communis & medium & 1 & $\pm 90 \%$ & coil compaction \\
\hline 20 & 65 & Ant.communis & giant & ॥ & $\pm 85 \%$ & no follow-up \\
\hline 21 & 35 & Ant.communis & small & 1 & $\pm 90 \%$ & no follow-up \\
\hline
\end{tabular}

\section{Technique}

A guiding catheter is advanced as distally as possible into thelarge vessels. Via Y-connections a microcatheter (we use Target's Fast-Tracker 10 and 18 microcatheters for this purpose) is introduced into the aneurysm by over the wire (micro guidewire) technique during constant flushing with heparinised saline. A very thin and floppy guidewire (Seeker light or others) is firstly introduced into the aneurysm and the microcatheter is then advanced into the lumen of the aneurysm. We steam-bend catheters into the shape of the distal arteries in relation to the aneurysm. The distal tip of the catheter should be in the central position of the aneurysm so as to allow the memory curl of the coil to take shape as soon as the coil's distal tip is advanced into the intraluminal space of the aneurysm. Theidea is to form an outer basket against the wall of the aneurysm in such a way that it forms a smooth surface without bulging too far into the parent vessel. After the first coil is in a proper position and the initial basket pattern forms, the coil can be detached. Two wires are attached to a $1 \mathrm{~m} /$ Amp generator (Target Therapeutics). (Figure 7) One electrical pole is connected to the stainless steel part of the GDC and the other one to the patient. (Figures $8 a \& 8 b)$

Each catheter has two distinct metal markers-one at the tip of the catheter and one $3 \mathrm{~cm}$ from the tip. The soldered area should be just out of the distal tip of the catheter before detachment is commenced, but once a few coils have been introduced into the aneurysm the distal tip inside the mesh of coils is not visible. Asstated above, a mark on the stainless wire coincides with the marker on the catheter at exactly the distance where the soldered part will be out of the catheter. Once the two markers are crossed or coincide detachment can

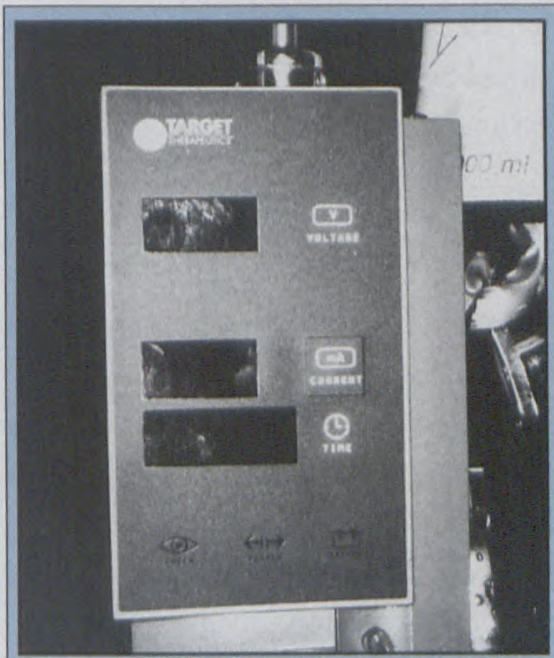

Figure 7: The generator manufactured by Target Therapeutics can be clamped to a drip stand. At the bottom the two wires with the negative and positive potentials can be seen.

commence. An alarm is triggered in the generator once the soldering is destroyed.

The great advantage of detachable platinum coils is the retractability of the coil. If a coil is misplaced before detachment it can be removed simply by direct removal.

Coils are stacked into the aneurysm until the basket is as tightly packed as possible from the larger outer to small inner 
from page 13

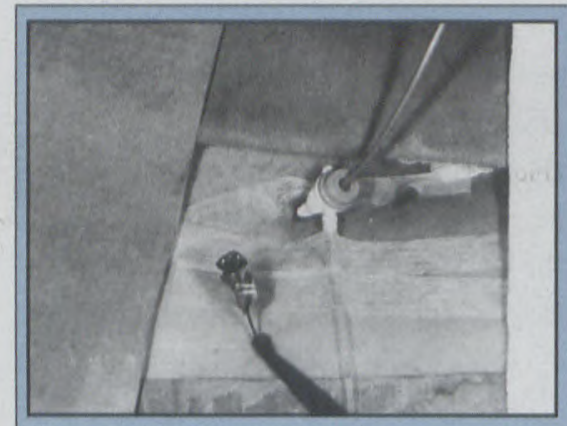

Figure $8 a$ \& b: The electrical wires are attached to the patient.

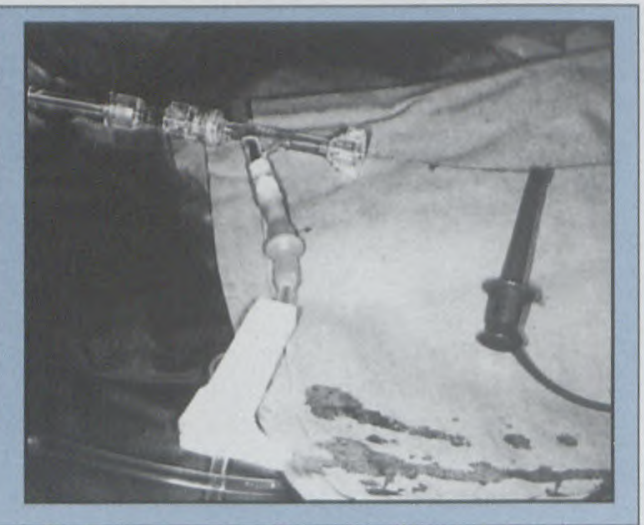

during the procedure. After the procedure the patient is monitored in the intensive care unit for 24 to 48 hours.

\section{Discussion}

The GDC treatment via the endovascular route is a new adjunct to the surgical approach to aneurysms. The indications for its use will vary from centre to centre as expertise and experience increase.

Coil compaction in the larger aneurysms is a problem and should be kept in mind.

In South Africa the cost of one coil is in the order of R3 500.00. A very large aneurysm can take up to 18 or more coils making the treatment expensive. Most small and medium aneurysms will take up to 5-6 coils.

Theidea of the treatment of aneurysms is to obtain vessel wall architectural restitution. Intima to intima as with surgical clipping, remains the golden standard. It is found that intimal proliferation over the coils at the surface of the parent vessel is absent (work in progress - reported in Val d'Isere France 1996) centrally but a fibroplastic response is seen at the edges of the neck of the aneurysm where some of the coil loops are covered with the latter.

Long term follow-up may demonstrate the increase of compaction. Newer coils which are softer may help in obtaining denser packing.
The application of stents over the ostiae is being researched at present, as well as the introduction of a polymer into the aneurysm once a few coils have been introduced.

\section{Complications}

In all the giant aneurysms, coil compaction wasseen. Thelarger the volume oneneeds to fill with coils the more dead space is possible between the loops of the separate wires. Three of the medium and none of the small ones had coil compaction. The tighter the initial "ball" of coils can be introduced theless probable the compaction would be. In somecentres soft coils are now being introduced in order to secure a tighter packing. Longer follow-up studies are needed to prove that this problem has been sufficiently dealt with by this means.

\section{Thrombo- embolism}

Two patients had thrombo-embolic sequelae.

One patient with a wide necked aneurysm at the confluence of the two vertebral arteries into the basilar artery developed a basilar artery thrombosis with a medullary infarct and died.

Another patient,following a carotid artery surgical procedure, had an embolisation of a small carotid artery aneurysm with GDC. The patient developed a middle cerebral artery thrombosis and stroke with motor aphasia and right hemiplegia.

Although both of these patients received heparin with an ACT of over 300 , they both developed thrombo-embolic phenomena.

\section{Vessel spasm}

Large and smaller vessel spasm has developed in a smaller percentage of our patients. We feel that calcium blockers during these procedures to be of the utmostimportance. blanket" to keep them warm and apply full cardiological and vascular monitoring 


\section{from page 14}

No aneurysm perforation or rebleed was reported in any of these patients.

\section{Conclusion}

With the advent of Guglielmi detachable coils a new chapter in the treatment of certain cerebral aneurysms has been introduced.

Development in this direction over the next years may well prove to be of major importance.

The costs involved can be a problem especially with the present exchange rate of the Rand to the USDollar.

The small and medium aneurysms with narrow necks seem to be the easiest to treat by this method while coil compaction appears to be present in the majority of giant aneurysms.
A team effort is absolutely necessary where the neurosurgeons, neuro-interventional radiologists, neurologists and anaesthetists work closely together as a team.

\section{References}

1.Taylor, PE: Delayed postoperative haemorrhage from intracranial aneurysm after craniotomy for tumor. Neu rology 1961;11,225-231.

2. Wieber, DO, Torner, JC and Meissner, I. Impact of Unruptured Intracranial Aneurysms on Public Health in the United States, Stroke, 1992; 23:10.

3.Weir, B. Aneurysms affecting the nervous system, Chapter2, 1987;19-54

4.MassoudTF, Guglielmi G, Vinuela F, Duckwiler GR. Saccular aneurysms in moyamoya disease: endovascular treatment using electrically detachable coils. Surg Neurol 1994;41(6):462-7,June

5.Ferguson G G: Physical factors in the initiation, growth, and rupture of human intracranial saccular aneurysms. J Neurosurg 1972;37:666-677.
6.Liepsch DW, Steiger HJ, Poll A, et al: Hemodynamic stress in lateral saccular aneurysms. Biorheology 1987;24:689-710

7.Kerber CW, Heilman CB:Flow in experimental berry aneurysms: method and model. ANNR 1983;4:374-377.

8.Sekhar LN, Sclabassi RJ, Sun M, et al: Intraaneurysmal pressure measurements in experimental saccular aneurysms in dogs. Stroke 1988;19:352-356.

9.Guglielmi G. Vinuela F. Dion J. Duckwiler G. Electrothrombosis of saccular aneurysms via endovascular approach. Part 2: Preliminary clinical experience. Joumal of Neurosurgery. 1991;75(1):8-14 July.

10. Hunt WE, Hess RM (1968): Surgical risk as related to time of intervention in the repair of intracranial aneurysms. J.Neurosurg.1968;28, 14-19.

\section{REVIEW}

\section{trompage 8 Cerebrovascular neuroimaging: which tests and when?}

specialities, a more cohesive interaction between the relevantspecialities is becoming a prerequisite for optimum patient evaluation. Never before has clinical acumen been more important in guiding the optimum use and non-use of the wide array of neurodiagnostic tools.

\section{Acknowledgement}

The author is most grateful to Professor JV Robbs for critical review of this manuscript and many helpful comments.

\section{References}

1.Von Reutern GM, von Buedingen HJ.Ultrasound Diagnosis in Cerebrovascular Disease. Doppler Sonography of the Extra and Intracranial Arteries, Duplex Scanning 1993, George ThiemeVerlag,New York.
2.AlexandrovAV, Bladin CF,Maggisano R, NorrisJW.Measuring carotid stenosis: a time for a reappraisal. Stroke 1993:24:1292-1296.

3. SteinkeW, Meairs S, Ries S, Hennerici M. Sonographic Assessment of Carotid Artery Stenosis. Comparison of Power Doppler Imaging and Color Flow Doppler Flow Imaging, Stroke 1996;27:91-94.

4. Griewing B, Morgenstern C, Driesner F, Kallwellis G, WalkerML, Kessler C. Cerebrovascular DiseaseAssessed by Color Flow and Power Doppler Ultransonography. Comparison With Digital Subtraction Angiography in Internal $\mathrm{Ca}$ rotidStenosis Stroke 1996;27:95-100.

5.American Academy of Neurology. Transcranial Doppler. Neurology 1990;40:680-681.

6.SchminkeU,Ries S, Daffertshofer M,Staedt U,Hennerici M. Patent Foramen Ovale:A Potential Source of Cerebral Embolism? Cendrouasc Dis 1995;5:133-138.

7.Siebler M,KleinschmidtA,Sitzer M,SteinmetzH,Freund H-J.Cerebral microembolism in symptomatic and asymptomatic high grade internal carotid artery stenosis. Neurology 1994,44615-618.

8. Frey Л, Greene KA, Khayata MH,Dean BL, HodakJA, Spetzler RF. Intrathrombus administration of Tissue Plasminogen Activator in Acute Cerebrovascular Occlusion. Angiology 1995;46:649-656.
9. Minematsu K, Li L, Fisher M, et al. Diffusion weighted magnetic resonance imaging: Rapid and quantitative detection offocal brain ischaemia. Neurology 1992;42:235-240.

10.SchwartzA. New Trends and Developments in Magnetic Resonancelmaging, Cerebrouasc Dis 1995;:139-144.

11.MinematsuK,LiL, Fisher M,SotakCH,DavisMA. Diffusion weighted MRI displays reversible and irreversible injury in atemporary ischaemicstrokemodel. Stroke 1992;23:145.

12.FurlanA.New MRITedmiquesfor Cenbrovasoular Diseases Seminar 261. AmericanAcademy of NeurologyAnnual Meeting Seattle, May 1995.

13. Hugg JW, Matson GB, Duyn JH, Maudsley AA, Weier MW.MR spectroscopicimaging of stroke. Stroke 1991;22:147.

14. LeclercX, Godefroy O, SalhiA, Lucas C, LeysD, PruvoJP. Helical CT for the Diagnosis of Extracranial Internal Carotid Artery Dissection.Stroke 1996;27:461-466.

15.Assessment of brain SPECT.Report of the Therapeutics and Technology Assessment Subcommittee of the AmericanAcademy of Neurology.Neurology 1996;46:278-285.

16. Baron JC, von Kummer R, del Zoppo GJ.Treatment of Acute Ischaemic Stroke. Challenging the Concept of a Rigid andUniversalTimeWindow.Stroke,26:2219-2221.

17. Alexandrov AV, Bladin CF, Ehrlich LE, Norris JW. Noninvasive Assessment of Intracranial Perfusion in Acute Cerebral Ischaemia.JNeurvimaging 1995;5:76-82. 